\title{
Efficient Localization in WSN Based on Structured Deployment of Anchor Nodes in DV-HOP
}

\author{
Ravneet Kaur $^{1}$ and Jyoteesh Malhotra ${ }^{2}$ \\ ECE Department, GNDU Regional Campus, Jalandhar, Punjab, India ${ }^{1,2}$ \\ ravneetkaur4141@gmail.com ${ }^{1}$,jyoteesh@gmail.com ${ }^{2}$
}

\begin{abstract}
Recent advancements in MEMSs technology, VLSI and Wireless Private Area Networks have contributed in developing enormous interest in wireless sensor networks by both academia and research community.WSN is formed by large number of small, cheap and low energy sensors that work together in monitoring regions. WSN find its application in many areas such as object tracking, disaster relief and smart environments. Most of these applications need location awareness of sensor nodes to estimate the origin of events. Localization has remained a challenge in successful implementation of WSN and so, it is a hotspot of research. Range free localization techniques are given lot of importance in recent research works because they suit well for low cost, low energy and less infrastructure requirements of WSN and also involve lesser computation complexity. DV$H O P$ is one of the range free techniques that have attracted researchers' interest. In this paper, DV-HOP algorithm is implemented by deploying anchor nodes in a structured way at various radio ranges. Structured deployment of anchors give more accurate location estimates than random deployment that was followed in basic DV-HOP, which has been demonstrated in a detailed manner by the simulation results.
\end{abstract}

Keywords: Localization; DV-Hop Algorithm; Localization Error.

\section{Introduction}

Wireless Sensor Networks (WSNs) [1] are large ad-hoc networks that are composed of tiny, cheap and low powered sensors interconnected wirelessly. These sensor nodes have perception, processing and communication ability that helps them to form a multi-hop self configured network. Mainly, the work of sensor nodes is to monitor the region in which they are deployed. In many application areas such as object tracking, environment monitoring, battlefield surveillance, it is required that monitoring messages from sensors should contain their location information [14].This is because; the information collected by the sensors will gain significant value only if the origin of the monitored event is determined from it. So, location of sensors can help in estimating spatial relationship between them and the starting point of monitored event. In WSN, some sensors with known location (GPS equipped) are deployed that further help other sensor nodes with unknown location to determine their location. Nodes with known location are referred as anchor nodes and nodes with unknown location are referred as unknown nodes The process of determining the physical coordinates of unknown sensor nodes using anchor nodes is known as self node-localization [2]. Localization techniques used in WSN can be categorized as-Range based techniques and Range Free techniques [9, 11]. Range based techniques need to use distance, angle, time of arrival, received signal strength measurements [10] to estimate the location of sensors. In contrast, Range free localization techniques need only connectivity information between the sensor nodes. So, Range free techniques overpower the range based techniques in terms of no extra hardware requirement, less power consumption, low cost and acceptable accuracy that are main constraints in WSN. 
Many Range free localization techniques, include Centroid [7], DV-HOP [4, 8], APIT [3] and MDS-MAP [5] have evolved in past years and many improvements are also done to increase their effectiveness. In this paper, we pay attention to Distance vector-HOP (DV-HOP) based localization technique. Major sources of error in DV-HOP are irregularly placed anchors due to which some sensors have very near anchors and some have anchors too far from them. This leads to unrealistic hop counts and hence, erroneous location estimation.

To enhance DV-HOP localization technique, improved methods have been reported. Qiqqian Huang et.al [12] proposed improved DV-HOP by placing some equally spaced anchors at the boundaries of network area and got better estimates of Average Hop Size and final location of nodes compared to random placement of anchors. Hongyang Chen et. al. [13] proposed IDV-HOP method that showed better localization accuracy when anchors were placed uniformly in the network rather than placed randomly. Yousi Zheng, et. al. [6] put forward a long range DV-HOP localization algorithm by using proper placement strategy of anchors to get more accuracy with lesser amount of anchor nodes in the network. Mohamad Ramazany et.al [15] proposed MDV-HOP localization algorithm in which anchors are placed manually rather than randomly. This regular placement helps in achieving more accurate results with increased coverage of anchor nodes.

In this paper, we will focus on structured deployment of anchors by implementing square deployment, uniform deployment and circular deployment strategies in the network area and compare them with random deployment strategy. Simulations have been done to choose the best strategy and optimum radio range to implement it.

The rest of paper is organized as follows: Section 2 explains the background followed by the methodology for simulations of DV-HOP algorithm in section 3. In Section 4, Simulations are shown and results are discussed. Finally, conclusion is drawn in Section 5.

\section{Background}

DV-HOP localization scheme was proposed by Niculescu and Nath [4] that depends on distance vector exchange method. It can be implemented using three steps:

Step 1. Each anchor node broadcasts the packet of data \{position, hop count, node id to its neighbor node. Each receiving node accepts the message from minimum hop count anchor and discards the others. Thus, all anchor nodes get minimum hop count from every anchor node. Then hop count plus one is forwarded to its neighboring nodes.

Step 2. Anchor nodes calculate the average size of hop count by using the information from step 1. This hop count value is flooded in controlled manner to all the sensor nodes which helps unknown nodes to calculate their distance from anchor nodes by multiplying hop size and minimum hop count value between them.

Step 3. When the unknown node gets three or more distances between anchor nodes, the position of unknown nodes can be estimated using Trilateration method [6].

\subsection{Performance parameters}

Effectiveness of DV-HOP localization technique is measured in terms of certain parameters such as:

\subsubsection{Localization Error}

Localization error is the difference between actual location of unknown nodes and calculated location of these nodes. Therefore, localization error is determined by Euclidean distance between actual and calculated values and is given by:

$$
E_{r}=\sqrt{\left(x-x^{\prime}\right)^{2}+\left(y-y^{\prime}\right)^{2}}
$$


Where $(\mathrm{x}, \mathrm{y})=$ actual coordinates of unknown nodes and $\left(\mathrm{x}^{\prime}, \mathrm{y}^{\prime}\right)=$ calculated coordinates of unknown nodes.

\subsubsection{Number of Anchors Used}

Number of anchor nodes used in a wireless sensor network should be minimum to meet the basic requirements of WSN i.e.

a) Hardware Requirement: Anchors are equipped with GPS devices. Thus, lesser the anchors lesser will be the Hardware setup of GPS required.

b) Cost: Efficiency of position of anchors using GPS comes with cost. So, lesser the anchors lesser will be the cost of network.

c) Energy Consumption: Lesser the number of anchors lesser will be the energy consumed by GPS receivers equipped on them.

So, only that localization algorithm is considered to be good which evaluates position of unknown sensor node with minimum error in presence of minimum anchor nodes.

\section{3. Simulation Methodology and Environment}

DV-HOP localization algorithm is a range free technique that uses distance vector exchange in terms of the hops between the unknown nodes and reference nodes to estimate the location of sensor nodes. Simulations are done assuming certain parameters and setup environment as shown below:

Table1. Simulation Setup

\begin{tabular}{|c|l|l|}
\hline S.no. & \multicolumn{1}{|c|}{ Parameters } & \multicolumn{1}{|c|}{ Specifications } \\
\hline 1. & Area & $100 \times 100$ square area \\
2. & Sensor nodes & static \\
3. & Total nodes & 100 \\
4. & Anchor nodes & 8 \\
5. & Unknown nodes & 92 \\
6. & Placement strategies of & Random, Square, Uniform, \\
& Anchor nodes & Circular \\
7. & Radio Ranges & $25-50 \mathrm{~m}$ \\
8. & Simulations done & 500 \\
\hline
\end{tabular}

Each simulation follows certain steps to estimate the location of unknown nodes i.e. using DV-HOP localization algorithm. It is shown in the form of flow chart figure 1. 


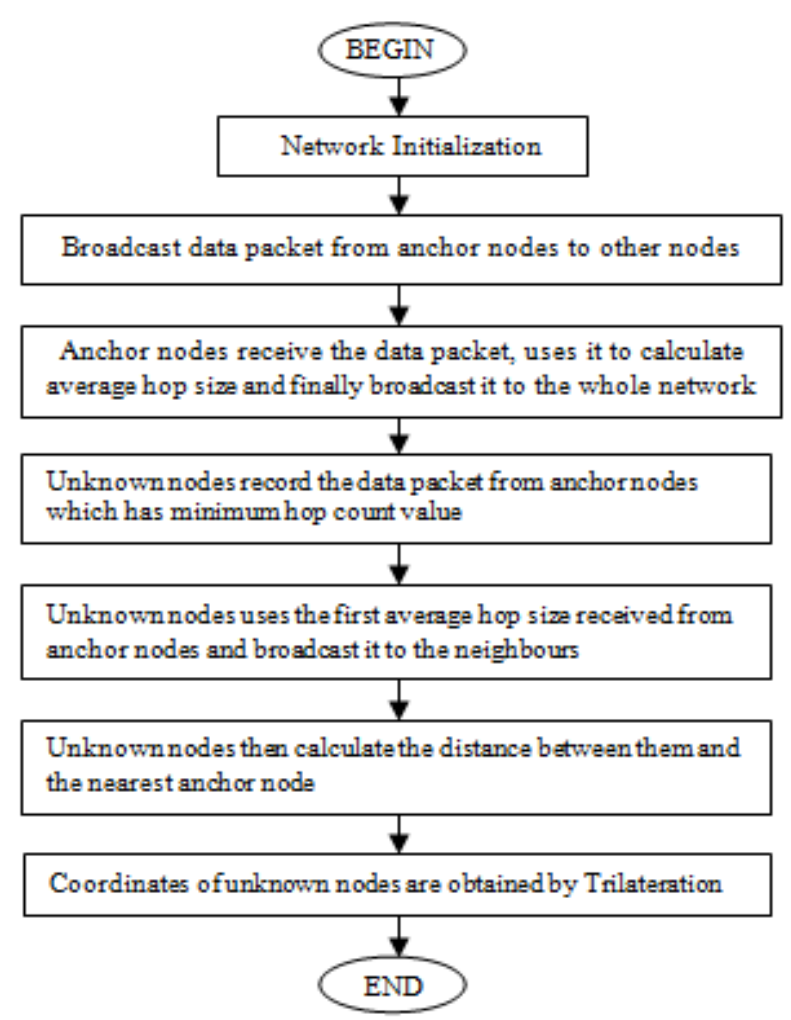

Figure 1. Flow Chart of DV-HOP Algorithm

\section{Results and Discussion}

Wireless sensor network considered during simulation has randomly distributed sensor nodes. But position of anchor nodes can be controlled manually. So, different placement strategies or topologies are implemented before implementation of DV-HOP localization algorithm that are:

(i)Random Deployment Strategy: Anchors are distributed in a random fashion within the network as shown in figure 1.

(ii)Square Deployment Strategy: Anchors are equally distributed on the edges of a square inside the network area as shown in figure 2.

(iii)Uniform Deployment Strategy: Anchors are distributed uniformly and equally spaced in a square area of network as shown in figure 3.

(iv)Circular Deployment Strategy: Anchors are distributed on the circumference of circle inside the network area as shown in figure 4.

These anchor deployment methods can be represented graphically as: 


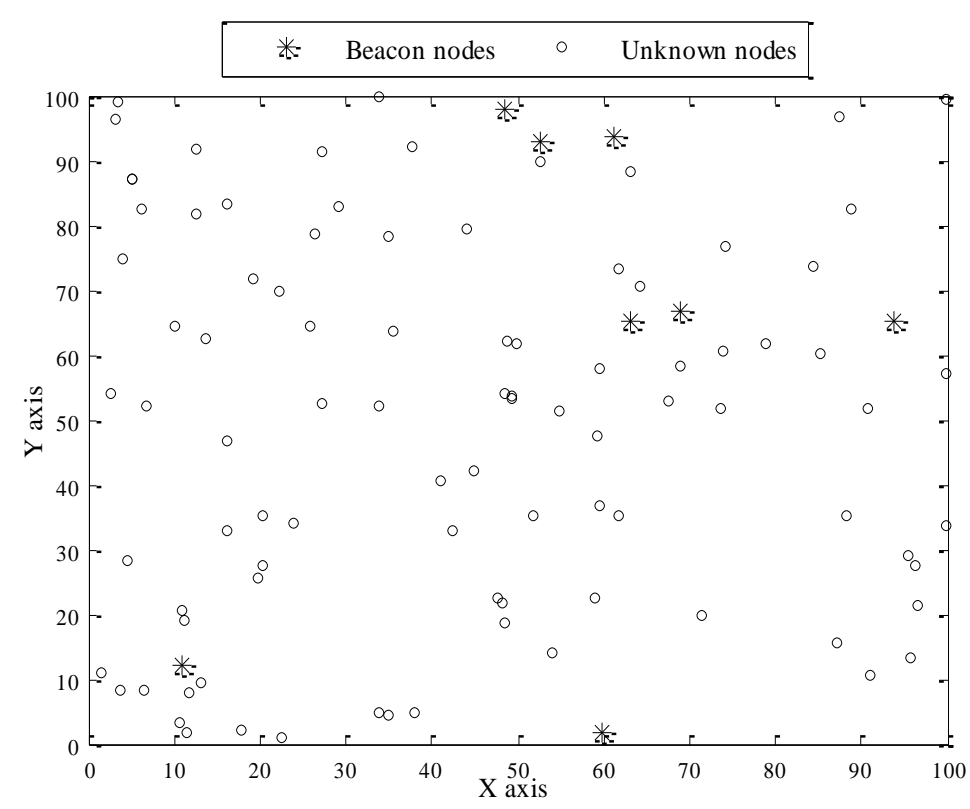

Figure 2. Random Deployment

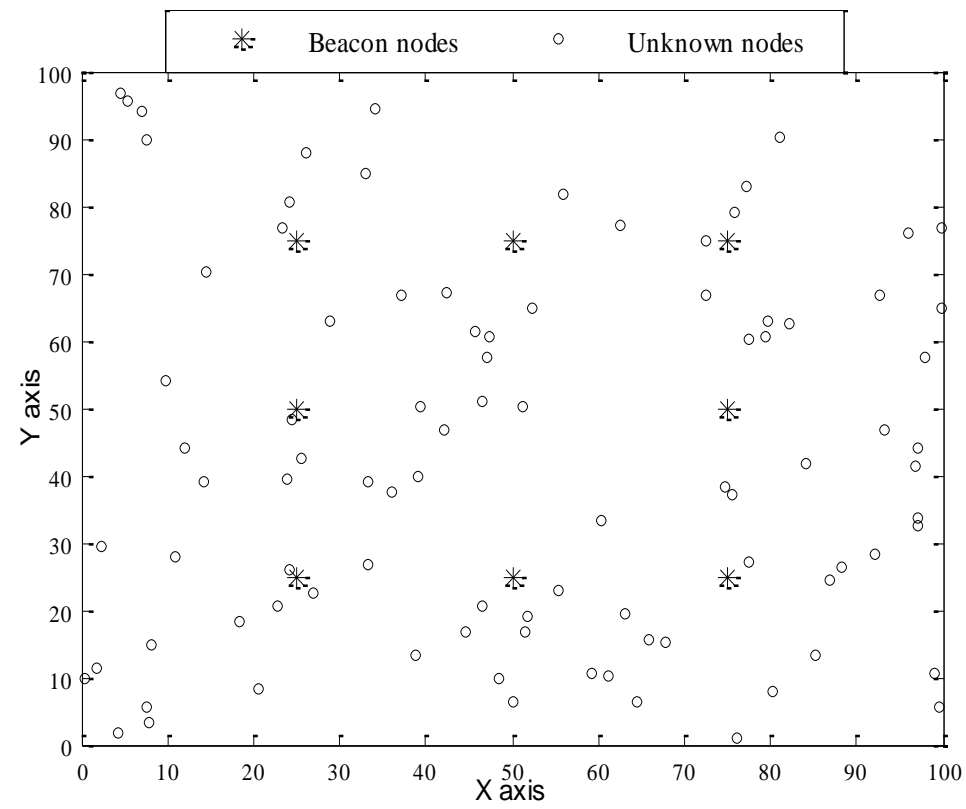

Figure 3. Square Deployment 


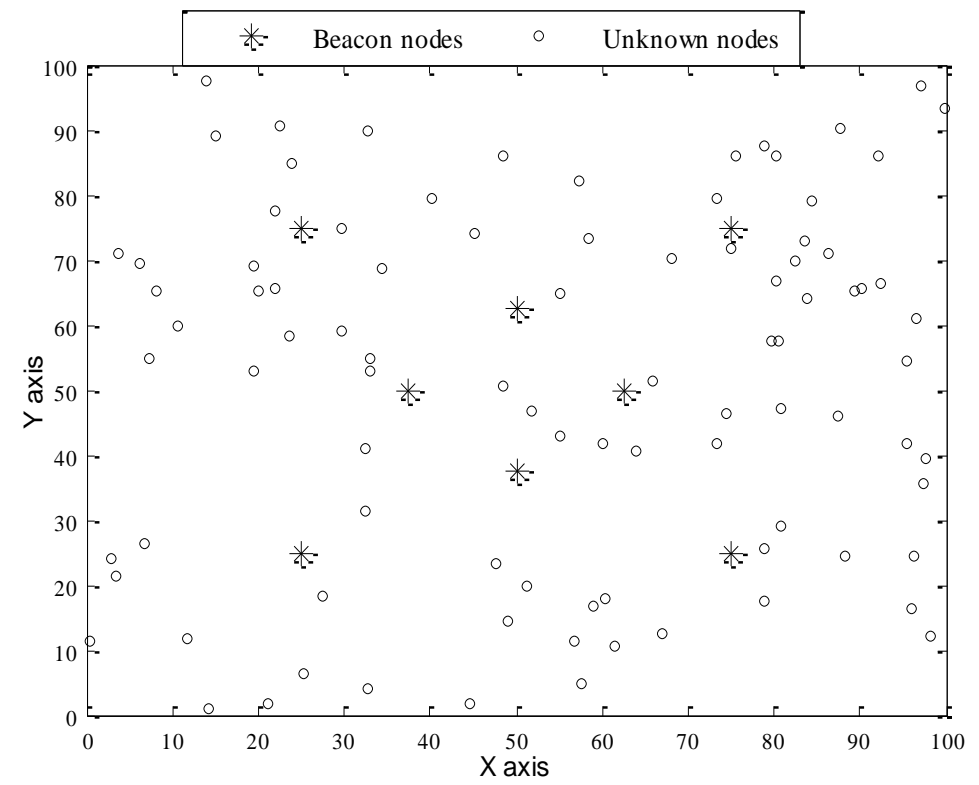

Figure 4.Uniform Deployment

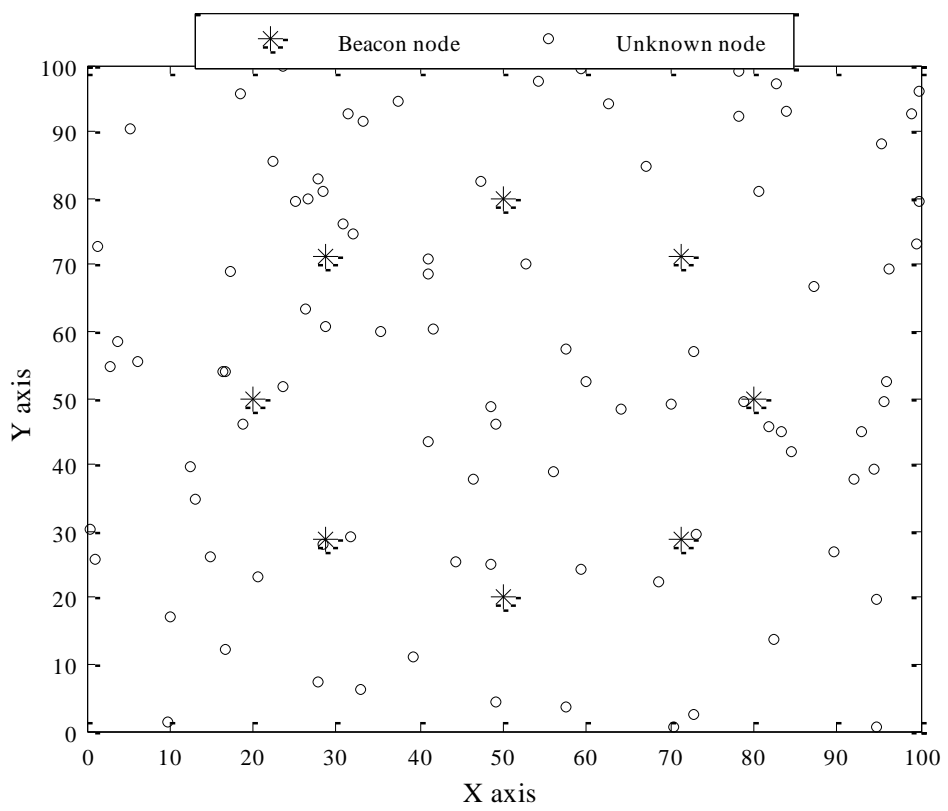

Figure 5. Circular Deployment

Performance of DV-HOP localization technique is evaluated on the basis of localization error for location of unknown nodes.

Detail analysis of error is done using first and second order of error statistical tools considering different deployment strategies of anchors in a network:

\subsection{Mean Localization Error}

Mean of Localization error is first order error statistical tool. It is given by:

$$
\overline{E_{r}}=\frac{\sum E_{r}}{n}
$$


Where $E_{\mathrm{r}}$ is Localization Error of each unknown node that is calculated using Eq. (1) and $\mathrm{n}$ is number of unknown nodes.

Mean of localization error is calculated for 500 simulations to compare between the various deployments strategies.

One major factor affecting the localization error is Radio range i.e. Range in which an anchor node can communicate with other nodes of the network.

More the radio range of an anchor node more will be the tolerance and coarser will be its location estimation. Thus, localization error increases with increase in Radio Range.

This is shown in error analysis shown in figure 6 and figure 7.

In circular deployment, we can have circles with Centre $(50,50)$ in $100 \times 100$ square area and radii $=25,30,35,40$ and 45 . So, for choosing appropriate radii, these circles are compared on the basis of mean localization error in each.

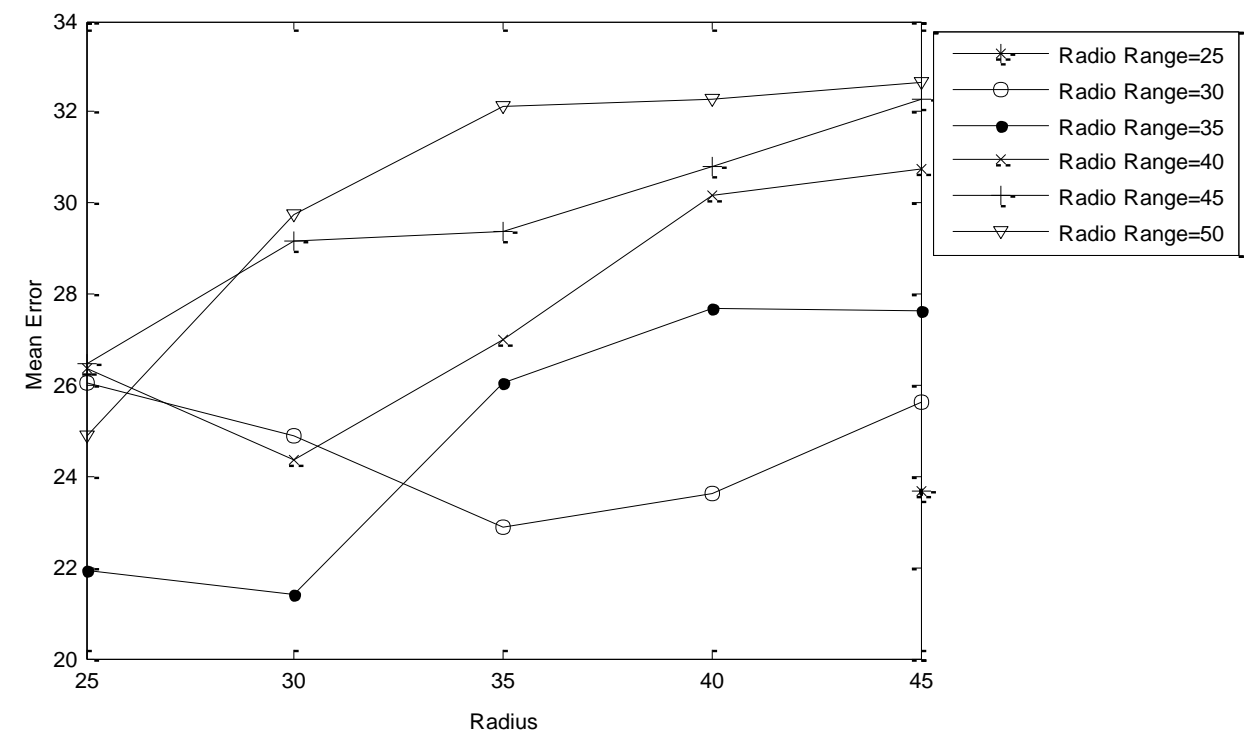

Figure 6. Mean Error at different Radii for Circular Deployment

Table2. Comparison of Circular Deployment on the basis of Radii of circle

\begin{tabular}{|c|c|c|c|c|c|c|}
\hline S.no. & Radio Range & Radius=25 & Radius=30 & Radius=35 & Radius=40 & Radius=45 \\
\hline 1 & 25 & $\mathrm{NaN}$ & $\mathrm{NaN}$ & $\mathrm{NaN}$ & $\mathrm{NaN}$ & 23.6972 \\
\hline 2 & 30 & 26.0363 & 24.8907 & 22.8868 & 23.6079 & 25.6521 \\
\hline 3 & 35 & 21.9220 & 21.3972 & 26.0711 & 27.6870 & 27.6219 \\
\hline 4 & 40 & 26.3583 & 24.3752 & 27.0145 & 30.1621 & 30.7714 \\
\hline 5 & 45 & 26.4622 & 29.1747 & 29.4032 & 30.8252 & 32.2573 \\
\hline 6 & 50 & 24.8905 & 29.7644 & 32.1366 & 32.2682 & 32.6325 \\
\hline
\end{tabular}

From Figure 6 and Table 2 we observe that:

At Radio Range $=25, \mathrm{Na} N$ i.e. not a number value is obtained at each radii except 23.6972.But still it is of less significance. Further we observe that, minimum error is obtained at radio range $=35$ for circle of radius $=30$ i.e. 21.3972 .

So, we will consider the circle with radius $=30$ for deploying 8 anchor nodes on its circumference. Hence, it will be used for comparison with other deployment strategies.

Comparing each of the four deployment strategy on the basis of mean localization error, we get the followed graph: 


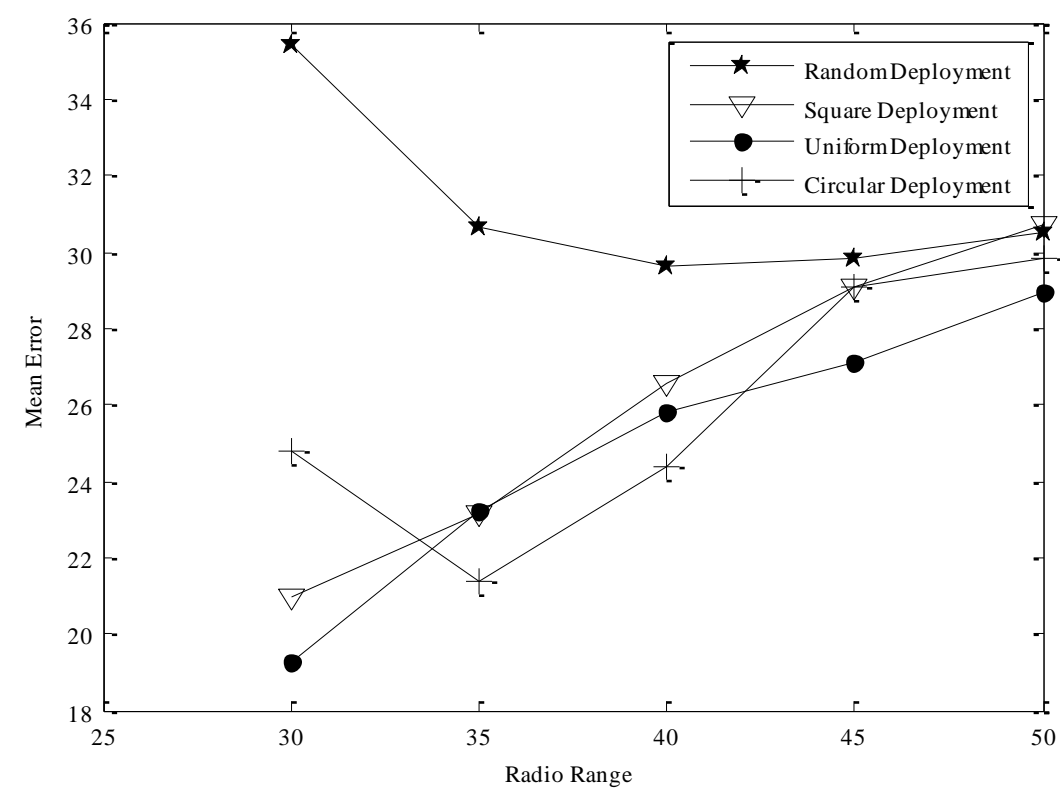

Figure 7. Mean Localization Error for Different Deployment Strategies

Table 3. Comparison of Different Deployment Strategies

\begin{tabular}{|c|c|c|c|c|c|}
\hline S.NO. & $\begin{array}{c}\text { Radio } \\
\text { Range }\end{array}$ & Random & Square & uniform & Circular \\
\hline 1 & 25 & $\mathrm{NaN}$ & $\mathrm{NaN}$ & $\mathrm{NaN}$ & $\mathrm{NaN}$ \\
\hline 2 & 30 & 35.3868 & 20.9803 & 19.2313 & 24.7932 \\
\hline 3 & 35 & 30.6316 & 23.1697 & 23.2353 & 21.3825 \\
\hline 4 & 40 & 29.6359 & 26.5252 & 25.8229 & 24.3851 \\
\hline 5 & 45 & 29.8122 & 29.1075 & 27.1135 & 29.0817 \\
\hline 6 & 50 & 30.4872 & 30.7032 & 28.9446 & 29.8276 \\
\hline
\end{tabular}

From figure 7 and Table 3, we observe that:

At Radio range $=25, \mathrm{NaN}$ values are obtained. So, no information can be drawn from this radio range.

Moving upward at Radio Range $=30$, minimum error $=19.2313$ is obtained for Uniform Deployment strategy.

Further at Radio Range $=35$, very closely related minima of error is obtained at 21.3825 for Circular Deployment strategy. After that increasing the Radio Range greatly increases the localization Error for all Placement Strategies.

\subsection{Cumulative Distribution Function (CDF)}

Cdf of a real valued random variable $\mathrm{X}$ is given by

$$
F_{X} x=P(X \leq x)
$$

Where, $\mathrm{P}(\mathrm{X} \leq \mathrm{x})$ represents the probability that the random variable $\mathrm{X}$ takes on a value less than or equal to $\mathrm{x}$. CDF of localization error is first order error statistical tool. It is used to know the probability of occurrence of mean error at each simulation under the graph. Here, we have evaluated CDF of all placement strategies are to get the information of spread of mean error for a specified deployment strategy. 


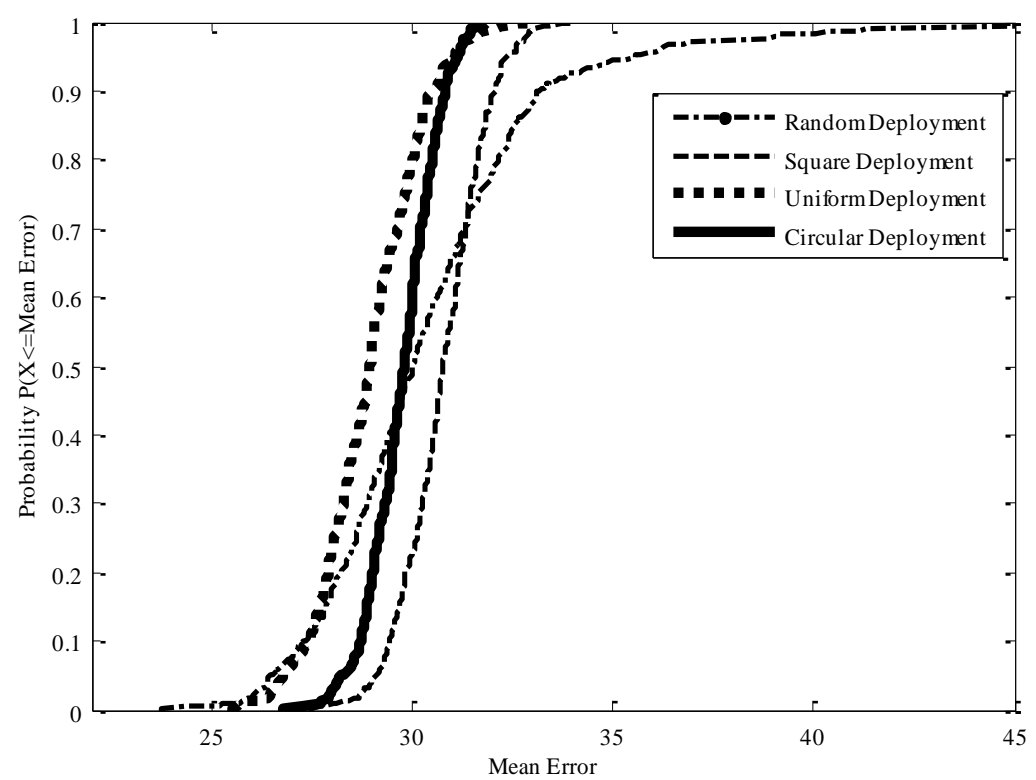

\section{Figure 8. Comparison of Various Deployment Strategies on the Basis of CDF}

From Figure 8, we observe that for Random Deployment strategy, there is $90 \%$ probability that mean localization error is less than or equal to 33.32

And for Square deployment strategy, there is $90 \%$ probability that Mean Localization error remains less than or equal to 32.07 and it also shows very steep slope as compared to Random Deployment Method. Further ,implementing Uniform Deployment in the network gives $90 \%$ probability that mean error will remain less than or equal to 30.55 and in circular deployment it will be very close value i.e. 30.85 .

So, from this first order statistical tool, we observed that Uniform and circular deployment performs better than Square and Random deployment. For deciding the best between these two we will study dispersion of error in these methods,

\subsection{Dispersion of Error}

The localization error for each node location estimation deviates from its mean value. The amount of variation is calculated through standard deviation. It is given by

$$
S . D .=\sqrt{\frac{1}{n-1} \sum_{r=1}^{n}\left(E_{r}-\overline{E_{r}}\right)^{2}}
$$

Where, Er is error at each node localization, $\overline{E_{r}}$ is mean error at each simulation and $\mathrm{n}$ is number of simulations. Standard deviation is a measure used to quantify amount of variation or dispersion of error values.

From figure 6 and 7, we observed that minimum error is achieved at radio range $=30$ and radio range $=35$ for uniform and circular deployment strategies. Further analyzing the error using second order error statistical tool i.e. standard deviation, we want to find the optimum deployment strategy and optimum radio range in which localization error deviates the least. More the value of standard deviation, more will be the dispersion of error and hence, more worsen is the performance of particular deployment strategy in given radio range. 


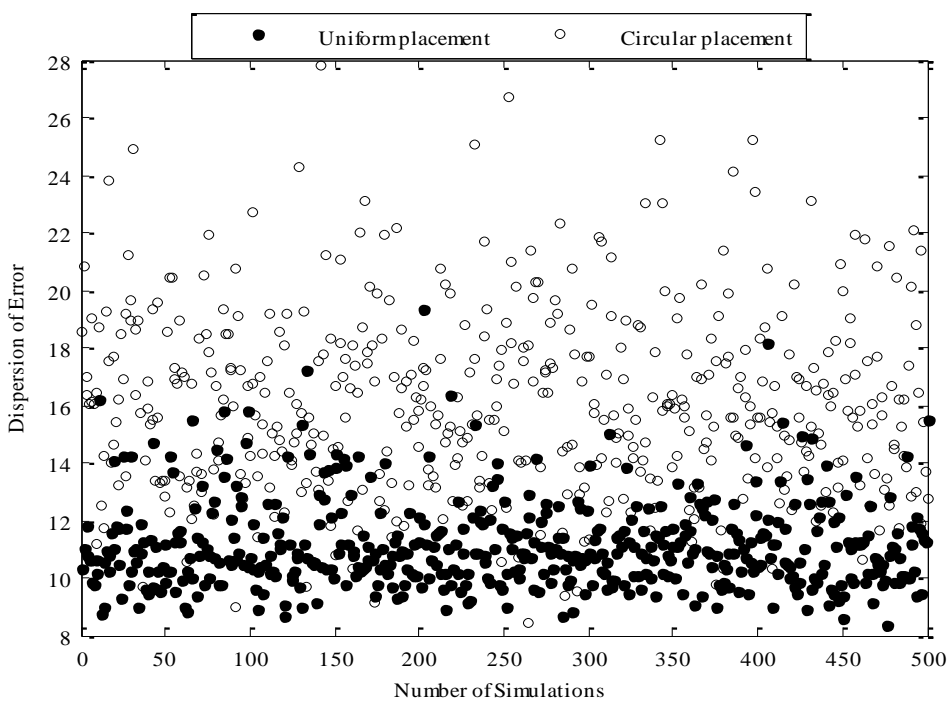

Figure 9. Dispersion of Error at Radio Range $=30$

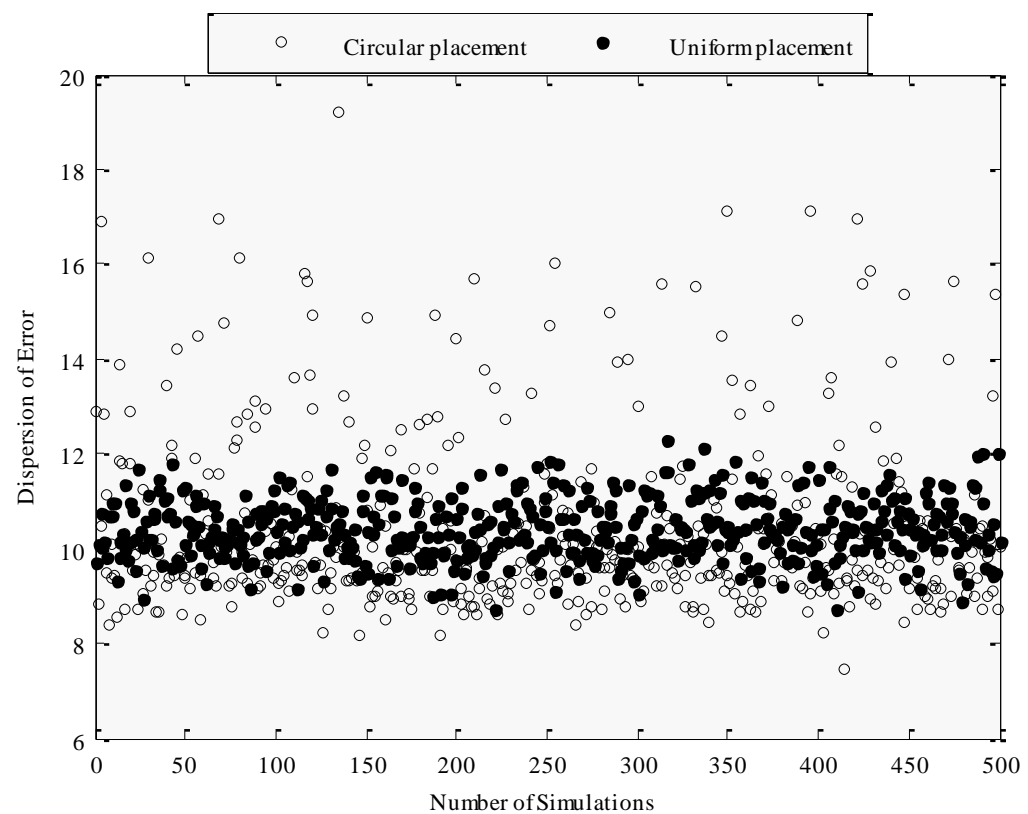

Figure 10. Dispersion of Error at Radio Range $=35$

From figure 9 and figure 10, it is clear that Uniform Deployment of anchors show lesser dispersion of error at both radio ranges of 30 and 35. Thus, Uniform Deployment of anchors shows best performance at required radio ranges with minimum dispersion of error.

\section{Conclusion}

This paper presents the basic DV-HOP localization algorithm in a different way by implementing it using four placement strategies of anchors in the network i.e. random deployment, square deployment, uniform deployment and circular deployment. The structured (square, uniform and circular) deployment of anchors greatly helps to improve the performance of DV-HOP localization Algorithm. With increase in radio range of 
Sensor nodes, the localization error also increases. So, detail analysis of error at different radio ranges is done from where we conclude that Uniform Deployment Strategy outperforms other deployment strategies in the optimum radio range of 30-35 m. Further, we can increase number of anchor nodes in structured deployment to get better results.

\section{References}

[1] J. Yick, B.Mukherjee and D. Ghosal, "Wireless sensor network survey", Computer Networks, vol. 52, no. 12 , (2008), pp. 2292-2330.

[2] M. Ramazany and Z. Moussavi, "Localization of Nodes in Wireless Sensor Networks by MDV-Hop Algorithm”, May 2012. J, Callaway, Phys. Rev. B 35 8723, vol. 2, no.5, (1987).

[3] T. He, C. Huang, B.M. Blum, J.A. Stankovic and T. Abdelzaher, "Range-free localization schemes for large scale sensor networks", in Proceedings of the 9th ACM Annual International Conference on Mobile Computing and Networking (MobiCom'03), (2003), pp. 81-95.

[4] D. Niculescu and B. Nath, "Ad-hoc positioning system", In IEEE Global Communications Conference, (GLOBECOM), (2001).

[5] Y. Shang, W. Ruml, Y .Zhang and M. Fromher, "Localization from mere connectivity in sensor networks" Fourth ACM International Symposium on Mobile Ad Hoc Networking and Computing (MobiHoc), (2003), Annapolis, MD.

[6] Y. Zheng, L. Wan, Z. Sun and Shunliang Mei, "A Long Range DV-Hop Localization Algorithm with Placement Strategy in Wireless Sensor Networks", IEEE (2008).

[7] N .Bulusu, J .Heidemann and D. Estrin, "GPS-less low-cost outdoor localization for very small devices", IEEE Personal Communications, vol. 7, pp 28-34, IEEE Personal Communications, vol. 7, (2000), pp. 28-34.

[8] D. Niculescu and B. Nath, "DV Based Positioning in Ad hoc Networks", Journal of Telecommunication Systems, (2003).

[9] Savvides, C.C. Han and M. Srivastava, "Dynamic fine-grained localization in ad-hoc networks of sensors", in Proceeding of the 7th ACM International Conference on Mobile Computing and Networking (MOBICOM, (2001), pp.166-179, Rome, Italy.

[10] F. Viani, L. Lizzi and P. Rocca,"Object Tracking through RSSI Measurements in Wireless Sensor Networks", Electronics Letters, vol. 44, no. 10, (2008), pp. 653- 654.

[11] W. Fubao, S. Long and R. Fengyuan, "Self-localization systems and algorithms for wireless sensor networks [J]", Journal of Software, vol. 16, no. 5, (2005), pp. 857-868.

[12] Q. Huang and S. Selvakennedy, "A range-free localization algorithm for wireless sensor networks", in Proc. of IEEE 63rd.Vehicular Technology Conference (VTC 2006-Spring), (2006), pp. 349-353.

[13] H. Chen, K. Sezaki, P. Deng and H.C. So, "An Improved DV-Hop Localization Algorithm for Wireless Sensor Networks", IEEE, (2008).

[14] Fundamentals of wireless sensor networks, theory and practice-Waltenegus Dargie (Technical university of Dresden, Germany), Christian Poellabauer( University of Notre Dame, USA)

[15] Wireless Sensor Networks, a Networking Perspective Edited by Jun Zheng, Abbas Jamalipour, A John Wiley \& Sons, Inc, Publication, (2009). 
International Journal of Future Generation Communication and Networking Vol. 9, No. 3 (2016) 